\title{
Factors Associated with Maternal Mortality at Gynecology and obstetrics Department in Departmental University Hospital Center of Borgou
}

\section{Salifou Badariatou, Atadé Sèdjro Raoul, Sidi Imorou Rachidi, Obossou Achille Awede, Sounouvi Ernest, Salifou Kabibou*}

Mother and Child Department, Faculty of Medicine (FM), University of Parakou (UP), Parakou, Benin

Email: *salifoukabibou@yahoo.com

How to cite this paper: Badariatou, S., Raoul, A.S., Rachidi, S.I., Awede, O.A., Ernest, S. and Kabibou, S. (2021) Factors Associated with Maternal Mortality at Gynecology and Obstetrics Department in Departmental University Hospital Center of Borgou. Open Journal of Obstetrics and Gynecology, 11, 1026-1036.

https://doi.org/10.4236/ojog.2021.118096

Received: June 18, 2021

Accepted: August 15, 2021

Published: August 18, 2021

Copyright $\odot 2021$ by author(s) and Scientific Research Publishing Inc. This work is licensed under the Creative Commons Attribution International License (CC BY 4.0).

http://creativecommons.org/licenses/by/4.0/

\begin{abstract}
Introduction: Maternal mortality is a robust indicator of human development. Most of it occurs in developing countries. Objectives: This study aims to determine the factors associated with maternal mortality in Gynecology and Obstetrics Department of Borgou Departmental University Hospital Center (CHUD-B). Study method: Our study took place at Gynecology and Obstetrics Department in Borgou Departmental University Hospital Center. This was a descriptive and analytical case-control study, covering a period of 7 years from January 1, 2012 to December 31, 2018. Results: Two hundred and twelve patients have been recruited consisting of 106 deceased women and 106 controls. The intra-hospital mortality ratio was 1100 deaths per 100,000 live births (LB). The mean age of deceased women was $27.06 \pm 6.45$ years with the extremes of 15 and 45 years. Direct obstetric causes were the most frequent causes of maternal death. They were dominated by immediate postpartum hemorrhage (32.93\%), followed by High Blood Pressure (26.83\%) and infections (17.07\%). The general condition of patients on admission (p-0.000) and inadequate management ( $\mathrm{p}-0.001)$ was associated with maternal mortality. Conclusion: Patients' general condition on admission and inadequate management was factors associated with maternal mortality.
\end{abstract}

\section{Keywords}

Maternal Mortality, Ratio, Direct Obstetric Causes, Indirect Obstetric Causes, Factors Associated 


\section{Introduction}

Pregnancy and childbirth place a woman at significant risk because of the unforeseeable complications to which they expose her [1]. The World Health Organization (WHO) estimates that 350,000 women die worldwide each year from complications related to pregnancy, childbirth or postpartum [2]. It is classified as a preventable fatality in contrast with other causes of death [3] [4] [5]. Despite the preventable nature of these deaths, a large number occur in developing countries representing ninety-nine percent (99\%) [6] [7]. According to World Health Organization (WHO), maternal mortality ratio was 239 per 100,000 live births in low-income countries compared to 12 per 100,000 live births in high-income countries in 2015 [8]. A reduction in maternal mortality of 75\% between 1990 and 2015 was envisaged in the fifth Millennium Development Goal (MDG) [9]. Progress has been made over the past decade [10]. As a result, the global maternal mortality ratio has been reduced by $43.9 \%$ [11]. This progress varied from region to region or continent to continent. In Benin, maternal mortality ratio fell from 600 in 1990 to 370 in 2010 and then to 320 in 2015 [12]. To achieve the Sustainable Development Goal (SDG) which is to reduce maternal mortality ratio to less than 70 per 100,000 live births in 2030 [11], it is necessary to identify the factors we could influence in order to better adapt the strategies to the local context. That is the subject of this article.

\section{Materials and Methods}

\subsection{Study Framework}

Our study took place in Gynecology and Obstetrics department of Borgou Departmental University Hospital Center (CHUD-B) in Parakou city.

\subsection{Study Methods}

\subsubsection{Type and Period of Collection}

This was an analytical case-control study covering a period of 7 years from January 1, 2012 to December 31, 2018.

\subsubsection{Study Population}

The study population consisted of all women admitted to the gynecology and obstetrics department of Borgou Departmental University Hospital Center CHUD-B during the study period for an obstetric cause.

\subsection{Inclusion Criteria}

Case: Any woman admitted to the service for any reason related to pregnancy or childbirth, who died according to WHO definition of maternal death and has been registered.

Control: Any woman who survived after being admitted to the service for any reason related to pregnancy or childbirth. 


\subsection{Exclusion Criteria}

All cases and controls with unusable medical records.

\subsubsection{Non-Inclusion Criteria}

- Any woman who died during pregnancy, childbirth or postpartum period outside of the service.

- Any woman who died in the service outside the context of pregnancy, childbirth or postpartum period.

- Death of a pregnant woman during pregnancy, childbirth or postpartum from a cause unrelated to pregnancy, childbirth or postpartum period.

\subsubsection{Matching Criteria}

The choice of controls was made as follows: We took either a woman of the same age or, failing that, whose age is more or less 2 years that of the case and who survived after her admission in the same month as the case for any reason in connection with the pregnancy.

\subsection{Sampling}

\subsubsection{Sample Size}

The sample size was calculated assuming an odds ratio between cases and controls of 7.5, taking 0.05 as a risk of first species and $20 \%$ ( $80 \%$ power) as a risk of second species. One (1) control per case and a mortality rate of $1.63 \%$ in 2018 were used as the basis for the calculation. It was thus obtained using Epi Tools epidemiological calculators with a minimum number of 102 cases and 102 controls.

\subsubsection{Sampling Technique}

An exhaustive census of all women meeting our inclusion and matching criteria has been conducted during our collection period. The sample size corresponded to the weight of control for a case.

\subsection{Data Collection}

\subsubsection{Collection Technique}

This involved a review of the individual files of cases and witnesses in order to gather the necessary information were gathered through a review of individual files of cases and controls.

\subsubsection{Data Collection Tools}

A previously drawn up counting sheet was used to collect information in the registers of death, childbirth, reports of the interventions of the operating theatre, reference sheets, individual patient records. The count sheet included sections on general information, obstetric history, complications and procedures experienced by women.

\subsection{Study Variables}

\subsubsection{Dependent Variable}

Maternal mortality. 


\subsubsection{Independent Variables}

- Sociodemographic characteristics: age, profession, residence, marital status.

- The medical, surgical and gyneco-obstetrical histories of patients.

- Pregnancy follow-up: Ante Natal Consultation (ANC), number of Ante Natal Consultation (ANC).

- Method of coming to care: by herself, referral.

- Referral: transport, referral form, venous access, referral center.

- Initial assessment of the patient on admission.

- Management: Diagnosis, complementary investigations, etiologic treatment, delay in treatment, causes of the delay.

- Characteristics relating to death: time between admission and death of patients, period of death, obstetric causes (direct and indirect), clinical audit performance.

\subsubsection{Data Processing and Analysis}

Data collected was entered into Epi data 3.1.fr software and processed using EPI INFO 7.2.0.1 software.

The odds ratio was used to measure the odds of dying or not. Pearson's chi-square test and Fisher's exact test were used as appropriate to measure association. The significance threshold was set at $5 \%$. Variables with a p-value less than or equal to $20 \%$ were introduced into a logistic model to distinguish the real factors associated with maternal death.

\section{Results}

From January 2012 to December 2018, 140 maternal deaths were recorded for 12,728 LB. The intra-hospital maternal mortality ratio (RMMIH) during that period was 1100 deaths per 100,000 LB. The evolution of these maternal deaths over time is shown on Figure 1.

Among those maternal deaths, 106 were the subject of our study; the others (34 cases) were excluded for lack of documentation. Results will therefore relate to 212 subjects including 106 (50\%) maternal deaths and 106 (50\%) controls.

The mean age in deceased patients was $27.06 \pm 6.45$ years with extremes of 15 and 45 years; $27.10 \pm 6.30$ years with extremes of 14 and 45 years in controls. The proportion of unaccompanied patients, patients referred without a referral form and without venous access was greater among the deceased women than among the controls with respective P-values at 0.008, 0.021 and 0.001 (Table 1).

There were more women who had not performed Ante Natal Consultation (ANC) among the deceased than among the controls with $\mathrm{p}<0.001$.

The number of miscarriages $(\mathrm{p}=0.029)$ and the number of children who died $(\mathrm{p}=0.04)$ were associated with maternal mortality (Table 2$)$.

There was no significant difference between patients in the death group and controls for indirect obstetric causes, $\mathrm{p}>0.05$.

Association reports between clinic and maternal deaths analyzed in Table 3 show a significant difference. 


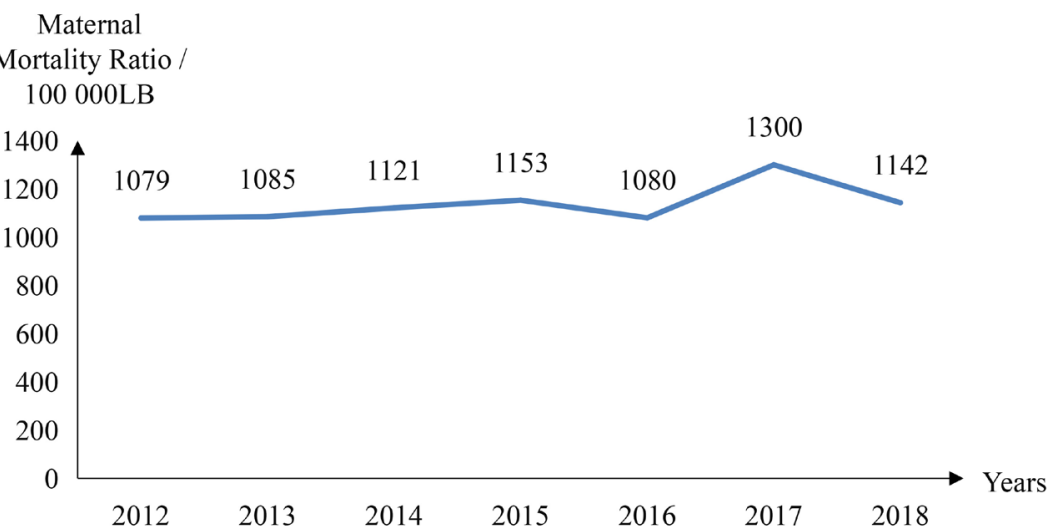

Figure 1. Distribution of the mortality ratio from 2012 to 2018 in gynecology and obstetrics department of CHUD-B in Parakou.

Table 1. Distribution of the 212 women studied according to a companion presence during the referral, referral form and venous access from 2012 to 2018 in gynecology-obstetrics department of CHUD-Borgou.

\begin{tabular}{|c|c|c|c|c|c|c|c|}
\hline & \multicolumn{2}{|c|}{ Cases } & \multicolumn{2}{|c|}{ Controls } & \multirow{2}{*}{ OR } & \multirow{2}{*}{$\mathrm{IC}_{95 \%}$} & \multirow{2}{*}{$\mathrm{p}$} \\
\hline & $\mathbf{n}$ & (\%) & $\mathrm{N}$ & $(\%)$ & & & \\
\hline \multicolumn{8}{|c|}{ Referrals (140 cases) } \\
\hline Accompanied & & & & & & & 0.008 \\
\hline Yes & 2 & 2.67 & 10 & 15.38 & 0.15 & $0.03-0.72$ & \\
\hline No & 73 & 97.33 & 55 & 84.62 & 1 & & \\
\hline Referral form & & & & & & & 0.021 \\
\hline Yes & 17 & 22.67 & 26 & 40.00 & 0.44 & $0.21-0.92$ & \\
\hline No & 58 & 77.33 & 39 & 60.00 & 1 & & \\
\hline Venous access & & & & & & & 0.001 \\
\hline Yes & 42 & 56.00 & 18 & 27.69 & 3.32 & $1.64-6.75$ & \\
\hline No & 33 & 44.00 & 47 & 72.31 & 1 & & \\
\hline
\end{tabular}

Table 2. Distribution of the 212 women studied according to pregnancy follow-up, obstetric history from 2012 to 2018 in the gynecology and obstetrics department of CHUD-Borgou.

\begin{tabular}{|c|c|c|c|c|c|c|c|}
\hline & \multicolumn{2}{|c|}{ Cases } & \multicolumn{2}{|c|}{ Controls } & \multirow{2}{*}{ OR } & \multirow{2}{*}{$\mathrm{IC}_{95 \%}$} & \multirow{2}{*}{$\mathbf{p}$} \\
\hline & $\mathrm{n}$ & (\%) & $\mathbf{N}$ & (\%) & & & \\
\hline Antenatal consultations & & & & & & & $<0.001$ \\
\hline Yes & 29 & 27.36 & 71 & 66.98 & 0.19 & $0.10-0.33$ & \\
\hline No & 77 & 72.64 & 35 & 33.02 & 1 & & \\
\hline \multicolumn{8}{|c|}{ Obstetrical histories } \\
\hline Number of miscarriage & & & & & & & 0.029 \\
\hline$<1$ & 85 & 80.19 & 97 & 91.51 & 0.38 & $0.16-0.86$ & \\
\hline$\geq 1$ & 21 & 19.81 & 9 & 8.49 & 1 & & \\
\hline Number of deceased children & & & & & & & 0.004 \\
\hline$<1$ & 78 & 73.58 & 95 & 89.62 & 0.32 & $0.15-0.69$ & \\
\hline$\geq 1$ & 28 & 26.42 & 11 & 10.38 & 1 & & \\
\hline
\end{tabular}


Table 3. Distribution of the 212 women studied according to the time between the onset of symptoms and admission to hospital, general and hemodynamics condition, vital distress and intensive care management from 2012 to 2018 in gyneco-obstetrics department of CHUD-Borgou.

\begin{tabular}{|c|c|c|c|c|c|c|c|}
\hline & \multicolumn{2}{|c|}{ Cases } & \multicolumn{2}{|c|}{ Controls } & \multirow{2}{*}{ OR } & \multirow{2}{*}{$\mathrm{IC}_{95 \%}$} & \multirow{2}{*}{$\mathrm{p}$} \\
\hline & $\mathbf{N}$ & (\%) & $\mathbf{N}$ & (\%) & & & \\
\hline $\begin{array}{l}\text { Time between the onset } \\
\text { of symptoms (hours) and } \\
\text { admission to hospital }\end{array}$ & & & & & & & $<0.001$ \\
\hline$<24$ & 52 & 49.06 & 68 & 64.15 & 0.16 & $0.07-0.40$ & \\
\hline $24-47$ & 18 & 16.98 & 28 & 26.42 & 0.14 & $0.05-0.37$ & \\
\hline $48-71$ & 3 & 2.83 & 3 & 2.83 & 0.21 & $0.04-1.28$ & \\
\hline$\geq 72$ & 33 & 31.13 & 7 & 6.60 & 1 & & \\
\hline General condition & & & & & & & $<0.001$ \\
\hline Good & 15 & 14.15 & 98 & 92.45 & 0.01 & $0.01-0.03$ & \\
\hline Poor & 91 & 85.85 & 8 & 7.55 & 1 & & \\
\hline Hemodynamic condition & & & & & & & $<0.001$ \\
\hline Stable & 67 & 63.21 & 90 & 84.91 & 0.31 & $0.16-0.59$ & \\
\hline Unstable & 39 & 36.79 & 16 & 15.09 & 1 & & \\
\hline Vital distress & & & & & & & $<0.001$ \\
\hline Yes & 48 & 45.28 & 17 & 16.04 & 4.33 & $2.27-8.25$ & \\
\hline No & 58 & 54.72 & 89 & 83.96 & 1 & & \\
\hline
\end{tabular}

The relation between treatment modalities and maternal deaths is summarized in Table 4.

Diagnostic errors and unavailability of complementary investigations on time were related to maternal deaths (Table 5).

Basing on the multivariate analysis, woman's general condition on admission $(\mathrm{p}<0.001)$ and third delay $(\mathrm{p}=0.002)$ were the factors influencing maternal mortality (Table 6).

\section{Discussion}

In our study, intra-hospital maternal mortality ratio (RMMIH) recorded during the 07 years was 1100 per 100,000 LB. Denakpo et al. [13] in 2010 in Cotonou found 1042 deaths per 100,000 LB as a ratio. Higher RMMIH was recorded in 2003 in Benin by Saizonou et al. [14] and in Niger by Garba et al. [15] in 2010 (2640 deaths per 100,000 LB). But, Saleh et al. [16] in Egypt, MacDorman et al. [17] in Colombia and Tej Delladji et al. [18] in Tunis reported lower ratios with respectively 79; 20 and 41 deaths per 100,000 LB. The RMMIH therefore greatly varies from country to country and from region to region. That great variability results from the difference in the socio-health organization level of countries or regions. 
Table 4. Distribution of the 212 women studied according to management adaptation to complementary investigation results, compliance with protocols and the third delay from 2012 to 2018 in the gynecology-obstetrics department of CHUD-Borgou.

\begin{tabular}{|c|c|c|c|c|c|c|c|}
\hline & \multicolumn{2}{|c|}{ Cases } & \multicolumn{2}{|c|}{ Controls } & \multirow{2}{*}{ OR } & \multirow{2}{*}{$\mathrm{IC}_{95 \%}$} & \multirow{2}{*}{$\mathbf{p}$} \\
\hline & $\mathbf{N}$ & (\%) & $\mathbf{n}$ & (\%) & & & \\
\hline $\begin{array}{l}\text { Adaptation of management } \\
\text { according to complementary } \\
\text { investigations results }\end{array}$ & & & & & & & 0.032 \\
\hline Yes & 15 & 14.15 & 6 & 5.66 & 2.75 & $1.02-7.38$ & \\
\hline No & 91 & 85.85 & 100 & 94.34 & 1 & & \\
\hline Etiologic treatment & & & & & & & $<0.001$ \\
\hline In compliance with the protocol & 55 & 51.89 & 103 & 97.17 & 0.96 & $0.51-1.82$ & \\
\hline Delay in performance & 31 & 29.25 & 0 & 0.00 & - & - & \\
\hline Non-compliant with the protocol & 20 & 18.87 & 3 & 2.83 & 1 & & \\
\hline Third delay & & & & & & & $<0.001$ \\
\hline Yes & 58 & 54.72 & 2 & 1.89 & 62.83 & $14.73-268.00$ & \\
\hline No & 48 & 45.28 & 104 & 98.11 & 1 & & \\
\hline
\end{tabular}

Table 5. Distribution of the 212 women studied according to the diagnosis rightness and the time taken to get complementary investigations results from 2012 to 2018 in the gynecology and obstetrics department of CHUD-Borgou.

\begin{tabular}{ccccccccc}
\hline & \multicolumn{9}{c}{ Cases } & \multicolumn{2}{c}{ Controls } & & \multirow{2}{*}{ OR } & IC95\% & p \\
\cline { 2 - 5 } & $\mathbf{n}$ & $(\%)$ & $\mathbf{n}$ & $(\%)$ & & & $<0.001$ \\
Diagnosis & & & & & & & \\
Right & 87 & 82.08 & 104 & 98.11 & 0.19 & $0.04-0.88$ & \\
Non-established & 10 & 9.43 & 0 & 0.00 & - & - & \\
$\quad$ Wrong & 9 & 8.49 & 2 & 1.89 & 1 & & $<0.001$ \\
Complementary & & & & & & & \\
investigations results & & & & & & & \\
Performed on time & 64 & 60.38 & 93 & 87.74 & 0.21 & $0.11-0.43$ & \\
Delay in performance & 42 & 39.62 & 13 & 12.26 & 1 & & \\
\hline
\end{tabular}

Table 6. Logistic model of factors associated with maternal mortality in multivariate analysis.

\begin{tabular}{cccc}
\hline Facteurs associated & OR Adjusted & IC $95 \%$ & $\mathrm{p}$ \\
\hline General condition & $\mathbf{6 2 . 9 7}$ & $1.91-208.10$ & $<0.001$ \\
Hemodynamic condition & 22.56 & $0.67-756.81$ & 0.082 \\
Vital distress & 0.05 & $0.00-0.74$ & 0.078 \\
Diagnosis & 0.28 & $0.01-15.34$ & 0.536 \\
Complementary investigations & 0.34 & $0.03-3.87$ & 0.382 \\
management adapted to the complementary investigations & 8.57 & $0.33-220.11$ & 0.194 \\
Etiologic treatment without protocols & 0.03 & $0.00-0.74$ & 0.032 \\
Third delay & 7.49 & $\mathbf{2 . 1 2 - 2 6 . 5 2}$ & $\mathbf{0 . 0 0 2}$ \\
Inadequate management & $\mathbf{0 . 1 0}$ & $\mathbf{0 . 0 3 - 0 . 3 7}$ & $\mathbf{0 . 0 0 1}$ \\
\hline
\end{tabular}


On univariate analysis, several factors are identified as being associated with maternal mortality. Among these factors, there is the place of residence. Women who lived in rural areas were twice more likely to die than those living in urban areas in this study, by contrast with Godefay et al. [19] who did not find a significant link between the living environment and maternal mortality. In Benin, women living in rural areas are housewives. As a result, pregnancies are not well followed and deliveries take place without qualified assistance. Thus, women who have not performed ANC are five times more likely to die than those who had at least four antenatal visits. For Yambare et al. [20] in Congo, the risk of death is three times higher among those who did not have a good follow-up. Nair et al. [21] and Bauserman et al. [22] reported that inadequate use of care is a contributing factor in maternal death and $20 \%$ to $40 \%$ of complications are detected during pregnancy follow-up. Pregnancy follow-up therefore appears to be a necessary strategic complement to reduce maternal mortality [23].

Referrals made without support from a health worker as companion contributed seven times more to maternal death. Under these conditions, the continuum of care is not ensured during transport even if a venous access has been put in place before evacuation. As a consequence, a venous access presence is not the assurance of a continuum of care. It only indicates the passage in a health structure before admission. Similarly, patients referred without documents providing information on their condition and the level of care received before the referral died twice as frequently.

In Congo [24] and Chad [25], when the delay is greater than 72 hours, it is often associated with maternal death; as Moyo et al. [26] reported with a significant link between delay in seeking care and maternal death. Death also occurred 4 times more in a context of vital distress.

Based on multivariate analysis, the woman's general condition at admission ( $p$ $<0.001)$ and the third delay $(\mathrm{p}=0.002)$ were the factors influencing maternal mortality. The third delay included inadequate management $(\mathrm{p}=0.001)$ which included delay in initiating aetiologic treatment and non-compliance with protocols $(\mathrm{p}=0.032)$. Inadequate management contributes 63 times more to maternal death. This situation reflects shortages in human resources, medico-technical equipment and emergency kits; all made worse by the frequent lack of blood supply. That third delay was found in significant proportions in series by Mgawdere et al. [25] (96.8\%) and that of Agan et al. [26] (48.5\%). Only the poor condition on admission and inadequate management were associated with maternal mortality after a multivariate analysis.

\section{Limit of the Study}

The type of case-control study made it possible to determine the factors associated with maternal mortality and to measure the risk of maternal death. However, it should be noted that this was a retrospective collection study and like all studies of this type, the present study is limited by the fact that it uses obstetric 
records that are often insufficiently completed in the context of extreme urgency. This limit means that certain variables can sometimes be missing or that their level. With regard to the methodology, we can say that the objective is reached that the results obtained are valid

\section{Conflicts of Interest}

The authors declare no conflicts of interest regarding the publication of this paper.

\section{References}

[1] Aguemon, T.C., Bagnan, T.A., Adisso, S., Houndeffo, T., Lagoye, G. and Takpara, I. (2015) Study of Maternal Deaths 3 Years before and 3 Years after the Free Cesarean Section in a University Hospital Setting in Benin. Journal de la Société de Biologie Clinique du Bénin, No. 22, 63-67.

[2] Alkassoum, I., Djibo, I., Hama, Y., Abdoulwahabou, A.M. and Amadou, O. (2018) Risk Factors for Intra-Hospital Maternal Mortality in the Region of Maradi, Niger (2008-2010): About a Retrospective Study Carried Out in the 7 Regional Maternities. Tropical Medicine and Health, 28, 86-91.

https://doi.org/10.1684/mst.2018.0770

[3] Pacagnella, C.R., Gecatti, G.J., Parpinelli, A.M., Sousa, H.M., Haddad, M.S., Costa, L.M., et al. (2014) Delays in Receiving Obstetric Care and Poor Maternal Outcomes: Results from Multicentre Cross-Sectional Study. BMC Pregnancy and Childbirth, 14, 159. https://doi.org/10.1186/1471-2393-14-159

[4] Flippi, V., Chou, D., Rosmans, C., Graham, W. and Say, L. (2016) Levels and Causes of Maternal Mortality and Morbidity. Reproductive, Maternal, Newborn, and Child Health: Disease Control Priorities, 2, 51-70. https://doi.org/10.1596/978-1-4648-0348-2 ch3

[5] Yaya, S. and Kadio, B. (2017) Overview of Maternal Mortality in Africa and the World. In: Yaya, S.H., Ed., Risking Death to Bring Life: Maternal and Child Health Policies and Programs in Africa Canada, Presses de l'Université Laval, Québec, $1-20$.

[6] Jat, R.T., Déo, R.P., Goicolea, I., Hurtig, A.-K. and San Sébastien, M. (2015) Socio-Cultural and Service Delivery Dimensions of Maternal Mortality in Rural Central India: A Qualitative Exploration Using a Human Rights Lens. Global Health Action, 8, 24976. https://doi.org/10.3402/gha.v8.24976

[7] Ambike, A., Kawade, V. and Ambike, D. (2019) Maternal and Fetal Outcome of Obstetric Emergencies in a Tertiary Care Rural Teaching Hospital-A Pilot Study. International Journal of Gynaecology and Obstetrics Research, 2, 49-56.

[8] Castillo, P., Hurtado, J.C., Martinez, M.J., Jordao, D., Lovane, L., Ismail, M.R., et al. (2017) Validity of a Minimally Invasive Autopsy for Cause of Death Determination in Maternal Deaths in Mozambique: An Observational Study. Public Library of Science Medicine, 14, e1002431. https://doi.org/10.1371/journal.pmed.1002431

[9] Hanson, C., Cox, J., Mbaruku, G., Manzi, F., Gabrysch, S., Schellenberg, D., et al. (2015) Maternal Mortality and Distance to Facility-Based Obstetric Care in Rural Southern Tanzania: A Secondary Analysis of Cross-Sectional Census Data in 226000 Households. The Lancet Global Health, 385, e387-e395. https://doi.org/10.1016/S2214-109X(15)00048-0

[10] Say, L., Chou, D., Gemmill, A., Tunçalp, Ö., Moller, A.-B., Daniels, J., et al. (2014) 
Global Causes of Maternal Death: A WHO Systematic Analysis. The Lancet Global Health, 2, e323-e333. https://doi.org/10.1016/S2214-109X(14)70227-X

[11] Alkema, L., Chou, D., Hogan, D., Zhang, S., Moller, A.-B., Gemmill, A., et al. (2016) Global, Regional, and National Levels and Trends in Maternal Mortality between 1990 and 2015, with Scenario-Based Projections to 2030: A Systematic Analysis by the UN Maternal Mortality Estimation Inter-Agency Group. The Lancet, 387, 462-474. https://doi.org/10.1016/S0140-6736(15)00838-7

[12] Le Fonds français Muskoka (2016) Rapport annuel FSP Muskoka année 4: For the Reduction of Maternal, Neonatal and Infant Mortality in Ten French-Speaking Countries of Africa and Haiti. Ministère des affaires étrangère française, OMS, UNICEF, UNFPA, ONU Femmes.

[13] Denakpo, J.L., Zoumenou, E., Hounkpatin, B., Aguemon, C., Lokossou, A., Gbegan, P., et al. (2010) Impact of Clinical Audits on the Quality of Emergency Obstetric and Neonatal Care at the Cotonou Lagoon Mother and Child Hospital in Benin. Journal de la Société Africaine Des Gynécologues-Obstétriciens, 11, 13-17.

[14] Saizonou, J., Ouendo, E.M. and Dujardin, B. (2006) Maternal Deaths Audit in Four Benin Referal Hospitals: Quality of Emergency Care Causes and Contributing Factors. African Journal of Reproductive Health, 10, 28-40. https://doi.org/10.2307/30032469

[15] Garba, M., Nayama, M., Alio, A.P., Holloway, M.L., Hamisu, B.S. and Salihu, H.M. (2011) Maternal Mortality in Niger: A Retrospective Study in a High Risk Maternity. African Journal of Medicine Science, 40, 393-397.

[16] Saleh, W.F., Ragab, W.S. and Aboulgheit, S.S. (2013) Audit of Maternal Mortality Ratio and Causes of Maternal Deaths in the Largest Maternity Hospital in Cairo, Egypt (Kasr Al Aini) in 2008 and 2009; Lessons Learned. African Journal of Reproductive Health, 17, 105-109.

[17] Mac Dorman, M.F., Declercq, E. and Thoma, M.E. (2017) Trends in Maternal Mortality by Socio-Demographic Characteristics and Cause of Death in 27 States and the District of Columbia. Obstetrics and Gynecology, 129, 811-818. https://doi.org/10.1097/AOG.0000000000001968

[18] Tej, D.R., Bougatef, S., Ben, S.F., Ben, M.N., Gzara, A., Gritli, I., et al. (2014) The 2010 Tunisian National Maternal Mortality Survey: About Tunis Data. La Tunisie Medicale, 92, 560-566.

[19] Godefay, H., Byass, P., Graham, W.J., Kinsman, J. and Mulugeta, A. (2015) Risk Factors for Maternal Mortality in Rural Tigray, Northern Ethiopia: A Case-Control Study. PLoS ONE, 10, e0144975. https://doi.org/10.1371/journal.pone.0144975

[20] Yambare, A. and Ibemba, G. (2017) Analysis of the Determinants of Pre-Partum Maternal Mortality in the Republic of Congo 2013-2015. HAL Archives-Ouvertes, $21 \mathrm{p}$.

[21] Nair, M., Knight, M. and Kurinczuk, J.J. (2016) Risk Factors and Newborn Outcomes Associated with Maternal Deaths in the UK from 2009 to 2013: A National Case-Control Study. An International Journal of Obstetrics and Gynaecology, 123, 1654-1662. https://doi.org/10.1111/1471-0528.13978

[22] Bauserman, M., Lokangaka, A., Thorsten, V., Tshefu, A., Goudar, S.S., Esamai, F., et al. (2015) Risk Factors for Maternal Death and Trends in Maternal Mortality in Low- and Middle-Income Countries: A Prospective Longitudinal Cohort Analysis. Reproductive Health, 12, 1-9. https://doi.org/10.1186/1742-4755-12-S2-S5

[23] Foumsou, L., Saleh, A., Kaïmba, O., Djongali, S., Djimté, N. and Mignagnal, K. (2014) Determinants of Maternal Mortality at the General National Reference Hos- 
pital of N'Djamena-Chad. La Revue scientifique du Tchad, 1, 1-7.

[24] Moyo, N., Makasa, M., Chola, M. and Musonda, P. (2018) Access Factors Linked to Maternal Deaths in Lundazi District, Eastern Province of Zambia: A Case Control Study Analysing Maternal Death Reviews. BMC Pregnancy and Childbirth, 18, 101. https://doi.org/10.1186/s12884-018-1717-1

[25] Mgawadere, F., Unkels, R. and van den Broek, N. (2016) Assigning Cause of Maternal Death: A Comparison of Findings by a Facility-Based Review Team, an Expert Panel Using the New ICD-MM Cause Classification and a Computer-Based Program (INTERVA-4). An International Journal of Obstetrics and Gynecology, 123, 1647-1653. https://doi.org/10.1111/1471-0528.13969

[26] Agan, T.U., Archibong, E.I., Ekabua, J.E., Ekanem, E.I., Abeshi, S.E., Edentekhe, T.A., et al. (2010) Trends in Maternal Mortality at the University of Calabar Teaching Hospital, Nigeria, 1999-2009. International Journal of Women's Health, 2, 249-254. https://doi.org/10.2147/IJWH.S11971 